\title{
Untangling Potential Links between Childhood Trauma and the Psychological Response to the COVID-19 Pandemic: A Prospective Study Testing Multiple Mediation
}

Stephanie V. Rek ( $\nabla$ s.rek@med.uni-muenchen.de)

LMU University Hospital Munich

Matthias A. Reinhard

LMU University Hospital Munich

Markus Bühner

LMU Munich

Daniel Freeman

University of Oxford

Kristina Adorjan

LMU University Hospital Munich

Peter Falkai

LMU University Hospital Munich

Frank Padberg

LMU University Hospital Munich

\section{Research Article}

Keywords: depression, anxiety, stress, loneliness, paranoia, psychological well-being, perceived stress, early adversity, multiple mediation

Posted Date: July 27th, 2021

DOI: https://doi.org/10.21203/rs.3.rs-742177/v1

License: (c) (i) This work is licensed under a Creative Commons Attribution 4.0 International License. Read Full License 


\section{Abstract}

Childhood maltreatment (CM)has been associated with adverse psychosocial outcomes during the pandemic, but the underlying mechanisms are unclear. In a prospective online study using baseline and 10-week follow-up data of 391 German participants, we applied multiple mediation analyses to test to what extent COVID-19 perceived stressors mediate the association between CM and later adverse psychosocial outcomescompared to established mediators of rumination and insecure attachment. We also explored the relative importance of different COVID-19 related stressors in predicting adverse psychological trajectories using elastic net regression. Results showed that CM was longitudinally associated with all adverse psychosocial outcome. COVID-19 perceived stressors, rumination, and insecure attachment mediated this relationship and full mediation was observed for the outcomes anxiety, stress and psychological well-being. COVID-19-related concerns about the future wasmost strongly and consistently associated with adverse psychosocial functioning. These findings provide preliminary evidence that COVID-19 perceived stressors, in particular concerns about the future, may be a key mechanismunderlying the development of adverse psychosocial outcomes in individuals with a CM history. Thus, COVID-19 perceived stressors may require a higher priority for prevention and treatment efforts in vulnerable groups. Our results warrant replication in more representative cross-cultural samples.

\section{Introduction}

It is obvious that the COVID-19 pandemic and its associated social restrictions places an exceptional strain on individuals leading to a deterioration of mental health and well-being world-wide (1). Specifically, the pandemic has resulted in unprecedented major stressors that can pose enormous psychological challenges including a virtual standstill of our public and private lives, anxieties about getting infected, the course of disease, and receiving appropriate medical care as well as but not limited to job uncertainties and financial difficulties. In a recent study, we could show that a greater impact of such COVID-19-specific stressors during the pandemic was associated with increased psychological difficulties in a German general population sample (2). Moreover, representative cohort studies comparing changes in individuals before versus in the first few weeks of the initial lockdowns have suggested significant increases in mental health symptomatology $(3,4)$, also summarised in a recent meta-analysis (5). In addition, longitudinal studies have identified heterogeneous trajectories of mental health symptomatology during the pandemic. Here, younger age, female sex, lower income levels, economic inactivity, and pre-existing mental health conditions have been associated with worse longitudinal psychological trajectories in terms of depression, anxiety, and loneliness (e.g., 6-11). This demonstrates the importance of inter-individual differences in mental health trajectories and emphasises that identification of important risk factors and accompanying underlying mechanisms is key, which could allow for targeted care or prevention approaches.

One group of individuals that may be particularly vulnerable to the effects of the COVID-19 pandemic on mental health are those who experienced childhood maltreatment (12). Childhood maltreatment (CM), which includes traumatic experiences of abuse and neglect, is arguably the most consistent 
transdiagnostic risk factor across psychiatric disorders and lower psychological well-being as shown in in multiple retrospective case-control and longitudinal studies (e.g., 13-19). In the context of the COVID-19 pandemic, CM exposed individuals showed greater mental health difficulties in terms of symptoms of anxiety, depression, and posttraumatic stress disorder (PTSD), compared to non-exposed individuals in initial cross-sectional (20-23) and longitudinal (24) studies. However, not all CM exposed individuals develop mental health difficulties in adulthood and, so far, little is known about the exact pathways through which CM leads to an increased mental health risk (14). In order to improve treatment or even prevent an adverse mental health cascade during the current pandemic, it is therefore crucial not only to identify vulnerable groups by environmental stratification based on CM criteria, but also to deepen our understanding of potential core mechanisms linking psychopathology to $\mathrm{CM}$.

Stress sensitisation by early exposure to $\mathrm{CM}$ has been proposed as a key transdiagnostic mechanism leading to the evolvement of later psychopathology (25-28). In the current pandemic, for instance, CM exposed individuals may be sensitised and particularly reactive to stress, which could lead to the perception that COVID-19-related stressors are particularly stressful. In turn, this could increase levels of adverse psychosocial outcomes. We are aware of only one longitudinal study amidst the current COVID19 pandemic that showed that perceived stress mediated the association of early life adversity and depressive symptom severity in adolescents (29). Yet, this study did not differentiate stressors specific versus unspecific to the COVID-19 pandemic. Further, it is unclear whether this mediation generalises to mental health conditions other than depression, and if it also occurs in adults. Finally, it is unclear to what extent perceived stress still plays a relevant mechanistic role when compared to established transdiagnostic mediators between $\mathrm{CM}$ and mental health such as rumination (e.g., 14,30-32) and insecure attachment $(33,34)$. This can be tested using multiple mediation analyses adjusting for important confounding factors such as age, sex, income, educational attainment, and pre-existing mental health conditions.

This prospective study in individuals from the general population aims at investigating the relationship between $\mathrm{CM}$ and subsequent psychopathology and psychological well-being as well as the relative mediation via COVID-19 perceived stressors, rumination, and insecure attachment. Based on previous research and the theoretical considerations described above, we hypothesised i) that CM is associated with more adverse psychosocial outcomes in terms of depression, anxiety, stress, loneliness, paranoia, and psychological well-being, and ii) that these associations are mediated by COVID-19 perceived stressors, rumination, and insecure attachment. Understanding the factors linking higher rates of mental health difficulties during the current pandemic to $\mathrm{CM}$ can inform the development of targeted prevention and psychosocial treatment efforts. Since not all COVID-19 stressors may be equally important in predicting adverse psychosocial outcomes, we further explored their relative importance.

\section{Methods}

\section{Participants and Procedure}


A longitudinal survey was conducted in adults $(18+$ years old) with varying levels of $\mathrm{CM}$ who were recruited online via social media platforms and university mailing lists. The secure online survey software (LimeSurvey) was used for assessments, which was set up using a forced response format to prevent missing data and questionnaire block randomisation to circumvent potential carry over effects. As a reimbursement participant could be included in a prize draw by entering their email address at the end of the survey. Recruitment and initial assessment took place between April 2020 - May 2021 with follow-up assessment 10 weeks after the initial assessment.

All participants provided informed consent prior to participation, the study was conducted in accordance with the Declaration of Helsinki (35) and approved by the Faculty of Medicine of the Ludwig-MaximiliansUniversity Research Ethics Committee [Project Number: 20-118].

\section{Measures}

\section{Exposure (at baseline) \\ Childhood Trauma Questionnaire (CTQ)}

CM was assessed by the CTQ self-report questionnaire that comprises five subscales: emotional, physical, and sexual abuse as well as emotional and physical neglect (36; German version: 37). Each subscale consists of five items, which are rated on a 5-point Likert scale ranging from 1 (not at all) to 5 (very much). Reversed items were recoded. The subscale scores were calculated to represent a total score. Scores on the total scale range from 25 to 125 with higher scores indicating more severe $\mathrm{CM}$. In previous research good psychometric properties of the questionnaire have been reported (36) and internal consistency of the total score at baseline was excellent in the present study (Cronbach's $a=0.92$ ).

\section{Proposed Mediators (at baseline)}

\section{COVID-19-specific stressor impact index}

The COVID-19-specific stressor impact index of the COVID-19 Pandemic Mental Health Questionnaire (CoPaQ) (38) was used to assess COVID-19 perceived stressors over the past two weeks. The subscale includes different COVID-19 stressors (e.g., quarantine/curfew, small accommodation/home-office, financial difficulties, childcare responsibilities, and physical health concerns), which are rated on a 5-point Likert scale ranging from 0 (not at all) to 4 (very much). The "not applicable" answer option was recoded as zero. Scores are summed to form a total score, which can range from 0 to 56 and higher scores indicate greater COVID-19 perceived stressors. Preliminary psychometric evaluation of this subscale was reported to be sound (39).

\section{Perseverative Thinking Questionnaire (PTQ)}

Rumination was assessed with the PTQ (40), which consists of 15 items rated on a 5-point Likert scale ranging from 0 (Never) to 4 (Almost always). Items on content-independent negative ruminative thinking 
can be summed to a total score, which can range from 0 to 60 . Higher scores indicate higher levels of ruminative thinking. Ehring et al. (40) reported good psychometric properties of the scale and we observed excellent internal consistency $(a=0.96)$.

\section{Relationship Styles Questionnaire (RSQ)}

We used the RSQ (41; German version: 42) to measure insecure attachment styles. The scale is comprised of 30 items, which are rate on a 5-point Likert scale ranging from 1 (not at all) to 5 (very much). Reversed items were recoded. Attachment avoidance and attachment anxiety were defined as proposed by Roisman et al. (43; Model 3A). At baseline, internal consistency estimates of the attachment avoidance and attachment anxiety subscales were good $\left(a_{\text {Avoidance }}=0.80 ; a_{\text {Anxiety }}=0.80\right)$

\section{Psychosocial outcome variables (at 10-week follow-up) Depression, Anxiety and Stress Scales (DASS-21)}

We used the DASS-21 to measure levels of depression, anxiety, and stress during the preceding week (41; German version: 42). Items are rated on a 4-point Likert scale of 0 (did not apply to me at all) to 3 (applied to me very much or most of the time). Subscale scores can each range from 0 to 28 , respectively, and higher scores indicate greater levels of psychopathology. In clinical and non-clinical samples good psychometric properties of the scales have been reported (46). In our study, internal consistency estimates ranged from acceptable to excellent for each subscale ( $a_{\text {Depression }}=0.93, a_{\text {Anxiety }}=0.79$, and $a_{\text {Stress }}=0.89$ ).

\section{Revised-Green et al Paranoid Thoughts Scale (R-GPTS)}

Paranoia over the past fortnight was assessed with the total score of the 18-item R-GPTS (44; German version: 45 ). Items are rated on a 5-point Likert scale ranging from 0 (not at all) to 4 (totally). Scores can range from 0 to 72; higher scores indicate higher levels of paranoia. Excellent psychometric properties of the scale have been reported (47). The German version was translated from the original English version following common guidelines for forward and backward translation (49). The final version was approved by one of the authors of the original version (D.F.). At T2, internal consistency of the total score was excellent $(\alpha=0.91)$.

\section{UCLA Loneliness Scale (UCLA-LS)}

Loneliness was assessed using the UCLA-LS (47; German version: 48), which includes 20 items rated on a 5-point Likert scale ranging from 1 (not at all) to 5 (totally). Items that were reversed were recoded. The average score was built to represent the mean, with higher scores indicating greater loneliness. Good psychometric properties of the scale have been reported in previous research (51). In our sample, internal consistency was excellent at the second assessment timepoint $(a=0.94)$.

\section{WHO (Five) Well-Being Index (WHO-5)}


Psychological well-being was assessed with the WHO-5 (49; German version: 50). Five items are rated on a 6-point Likert scale ranging from 0 (at no time) to 5 (all of the time). Items were summed to represent the total score, which can range from 0 to 25 , higher scores indicate greater well-being. Sound psychometric properties of the scale have been reported in previous research (54). In our sample, internal consistency was excellent at follow-up $(a=0.90)$.

\section{Statistical analyses}

All analyses were conducted in R version 4.0 .0 (55) with packages psych (version 1.8.12; 53), lavaan (version 0.6-3.1295; 54), and glmnet (version 4.1-1; 55).

First, bivariate Pearson's correlation coefficients and Chi-square tests $\left(\chi^{2}\right)$ were conducted to test associations between variables of interest.

Second, multiple mediation analyses using the maximum likelihood estimator were performed. CM was used as predictor variable and depression, anxiety, stress, loneliness, paranoia, and psychological wellbeing as outcome variables in separate mediation analyses. Mediator variables of COVID-19 perceived stressors, rumination, and attachment (anxious- and avoidant) were included simultaneously in the analyses and were allowed to correlate with each other. Standard errors were calculated using 10,000 bootstrap samples because some variables did not fully adhere to a normal distribution. We report biascorrected $95 \%$ bootstrapped confidence intervals (Cl) for the total (c), direct (c'), and indirect effects. Subdivision of the total indirect effect to specific indirect effects allowed comparisons of the standardised effect sizes of each mediator. Standardised ordinary least squares regression coefficients are reported for all paths. To account for potential influences of age, sex, income, educational attainment, and pre-existing mental health conditions, variables were included as covariates in each multiple mediation model. Of note, since multiple comparisons were performed we controlled for false positive rate by using the Benjamini-Hochberg procedure (59).

Finally, to explore the relative importance of each of the different perceived COVID-19 stressors contributing to the total COVID-19 stressor index, we performed elastic net regression analyses (60) to explore the relative predictive value of individual stressors for adverse psychosocial functioning. This technique is an extension of ordinary least squares regression that better accounts for collinearity between baseline predictors and simplifies the statistical model using regularisation. It includes the two hyperparameters a (tuning parameter of 0 to 1 , which controls the type of shrinkage and, thus, the estimation method) and $\lambda$ (penalty parameter of 0 to 1 , which controls the amount of shrinkage with higher values leading to greater penalisation). The optimal hyperparameter combination was determined using grid search by selecting the model with smallest root mean squared error (RMSE) within 10-fold cross-validation. Of note, regularisation with $a=1$ equals Least Absolute Shrinkage and Selection Operator (LASSO) regression (61) and a = 0 equals Ridge (62) regression; a values between 0 to 1 reflect the relative balance between the two regression models. Elastic net regression models were controlled for age and sex. 


\section{Results}

\section{Sample characteristics}

Six hundred sixty-eight participants completed the survey at baseline (T1) and 429 at the 10-week followup assessment (T2). To ensure high data quality, we excluded participants at baseline who answered more than 1 bogus item incorrectly (e.g., not checking "very much" for the item "Please, indicate 'very much"') ( $n=58)$. In addition, participants with response times less than 25 minutes at baseline $(n=8)$ and less than 15 minutes at follow-up $(n=8)$ were excluded, which were deemed unlikely response times on the basis of personal experiences and response time descriptive statistics (baseline response time: median = $48 \mathrm{~min}$, $1 \mathrm{st}$ quartile $=38 \mathrm{~min}$, $3 \mathrm{rd}$ quartile $=61 \mathrm{~min}$; follow-up: median $=23 \mathrm{~min}$, 1 st quartile $=29$ min, 3 rd quartile $=40 \mathrm{~min}$ ). We also removed 30 participants who did not have a matching id variable between baseline and follow-up assessment, which resulted from a rare failure of the software. Taken together, this led to a final sample of 391 individuals $(77.43 \%$ females) on which analyses are based (age: mean $=30.99$, standard deviation[sd] $=11.52$ ). The final sample consisted of $91.82 \%$ participants who indicated German nationality, $47.83 \%$ were single (see Table 1 for more demographic and clinical characteristics). Of note, participants only completing the baseline assessment did not differ significantly from the follow-up sample in terms of age, sex, nationality, employment status, marital status, and preexisting mental health conditions ( $p>0.05$; see Supplementary Table 1 ). 
Table 1

Baseline characteristics of analytic sample

\begin{tabular}{|ll|}
\hline & Descriptive statistics \\
\hline Sample size, $n$ & 391 \\
\hline Age, mean (SD) & $30.99(11.52)$ \\
\hline Women sex, $n$ (\%) & $303(77.49)$ \\
\hline Employment status, $n$ (\%) & \\
\hline Full-time employed & $88(22.51)$ \\
\hline Part-time employed & $63(16.11)$ \\
\hline Self-employed & $14(3.58)$ \\
\hline Student & $185(47.31)$ \\
\hline Retired & $7(1.79)$ \\
\hline Caregiver & $0(0)$ \\
\hline Not employed & $11(2.81)$ \\
\hline Other & $23(5.88)$ \\
\hline Freely disposable money per month, $n(\%)$ & \\
\hline$<100 €$ & $28(7.16)$ \\
\hline $100-250 €$ & $84(21.48)$ \\
\hline $250-500$ & $111(28.39)$ \\
\hline $500-1000$ & $85(21.74)$ \\
\hline$>1000$ & $83(21.23)$ \\
\hline Educational attainment, $n(\%)$ & $33(18.67)$ \\
\hline Primary school & $352(90.03)$ \\
\hline Secondary school & \\
\hline A-levels & \\
\hline Self-reported lifetime diagnoses, $n(\%)$ & $(6.97)$ \\
\hline Number of diagnoses & \\
\hline 0 & \\
\hline 1 & \\
\hline
\end{tabular}




\begin{tabular}{|ll|}
\hline & Descriptive statistics \\
\hline 3 & $40(10.23)$ \\
\hline$>=4$ & $13(3.32)$ \\
\hline Diagnostic categories & $4(1.02)$ \\
\hline Depressive Disorders & \\
\hline Bipolar Disorders & $90(23.02)$ \\
\hline Psychotic Disorders & $3(0.77)$ \\
\hline Anxiety Disorders & $0(0)$ \\
\hline Post-Traumatic Stress Disorder & $45(11.51)$ \\
\hline Obsessive-Compulsive and Related Disorders & $7(1.79)$ \\
\hline Eating Disorders & $18(4.60)$ \\
\hline Substance-Related and Addictive Disorders & $4(1.02)$ \\
\hline Attention-Deficit/Hyperactivity Disorder & $9(2.30)$ \\
\hline Somatoform Disorders & $2(0.51)$ \\
\hline Personality Disorders & $9(2.30)$ \\
\hline Autism Spectrum Disorder & $3(0.77)$ \\
\hline Dementia & $0(0)$ \\
\hline
\end{tabular}

\section{Multiple mediation models}

Descriptive statistics of proposed exposure, mediator, and outcome variables can be found in Table 2. As a prerequisite for mediation analyses, we observed bivariate correlations between all proposed exposure and mediator variables (path a), mediator and outcome variables (path b), and exposure and outcome variables (path c) as shown in Supplementary Table 2 and Supplementary Table 3. For example, COVID19 perceived stressors were significantly associated with the predictor $\mathrm{CM}(r=0.26, p<0.01)$ and the criterion depression severity $(r=0.35, p<0.01)$. Rumination was significantly related to the predictor $(r=$ $0.27, p<0.01)$ and criterion variable $(r=0.50, p<0.01)$. Attachment anxiety was also significantly linked to $\mathrm{CM}(r=0.25, p<0.01)$ and depression severity $(r=0.32, p<0.01)$, as was attachment avoidance $\left(r_{C M}\right.$ $\left.=0.32 ; p<0.01 ; r_{\text {Depression }}=0.34, p<0.01\right)$. Lastly, depressive symptom severity was significantly associated with $\mathrm{CM}$ severity $(r=0.35 ; p<0.01)$. 
Table 2

Descriptive statistics of exposure, proposed mediator, and psychosocial outcome variables

\begin{tabular}{|llll|}
\hline & Mean (SD) & Range & IQR \\
\hline Exposure variable & & & \\
\hline T1 Childhood Maltreatment (CTQ) & $37.55(12.93)$ & $25-100$ & $29-43$ \\
\hline Proposed Mediators & & & \\
\hline T1 CoVID-19 Perceived Stressors (COPAQ) & $13.63(8.95)$ & $0-47$ & $7-20$ \\
\hline T1 Rumination (PTQ) & $27.70(14.00)$ & $0-59$ & $17-38$ \\
\hline T1 Anxious attachment & $2.13(0.91)$ & $1-5$ & $1.40-2.8$ \\
\hline T1 Avoidant attachment & $2.64(0.77)$ & $1-4.50$ & $2-3.13$ \\
\hline Criterion variables & & & \\
\hline T2 Depression (DASS-21) & $11.47(10.75)$ & $0-42$ & $4-16$ \\
\hline T2 Anxiety (DASS-21) & $5.86(6.86)$ & $0-36$ & $0-8$ \\
\hline T2 Stress (DASS-21) & $12.75(9.77)$ & $0-40$ & $4-20$ \\
\hline T2 Loneliness (UCLA-LS) & $2.19(0.72)$ & $1-4.45$ & $1.55-2.70$ \\
\hline T2 Paranoia (R-GPTS) & $8.33(10.06)$ & $0-57$ & $1.5-11$ \\
\hline T2 Well-being (WHO-5) & $12.21(5.71)$ & $0-25$ & $7.50-17$ \\
\hline Note. SD = standard deviation. IQR = inter quartile range. & & \\
\hline
\end{tabular}

In separate multiple mediation models and as indicated by the total indirect effect, we observed evidence supporting overall mediation of effects of $\mathrm{CM}$ on the different outcome variables (i.e., depression, anxiety, stress, paranoia, loneliness, and psychological well-being) via COVID-19 perceived stressors, rumination, and attachment (anxious and avoidant) (see Table 3 and Fig. 1). Specific indirect effects of the proposed mediators were significant for COVID-19 perceived stressors, rumination, and attachment avoidance across outcome variables. Attachment anxiety did not contribute additionally to the indirect effect in five out of six separate multiple mediation analyses; except paranoia. Full mediation between $\mathrm{CM}$ and anxiety as well as stress was observed as the direct effect ( $\left.c^{\prime}\right)$ was no longer significant after inclusion of the proposed mediators. Partial mediation was observed for the relationship between CM and depression, paranoia, loneliness, and psychological well-being since the direct effect remained significant. This suggests that our proposed set of mediators did not fully explain this relationship. Of note, all results from multiple mediation analyses were adjusted for the potential confounders age, sex, income, educational attainment, and pre-existing mental health conditions and the mediators were allowed to correlate with each other. When applying alpha correction for multiple testing, results remained largely 
unchanged but the specific indirect effect of attachment avoidance was no longer significant for the outcomes stress and paranoia. Moreover, full mediation was observed for psychological well-being (see Table 3). 
Table 3

Multiple mediation models with standardised bootstrap intervals

$\begin{array}{llllllll}\text { DV } & \begin{array}{l}\text { Std. point } \\ \text { estimate }\end{array} & \text { SE } & \text { P } & \text { Pcorrected } & \text { Cl } & \text { Lower } & R^{2} \\ & & & & & \text { Upper } & \end{array}$

\section{Depression}

\begin{tabular}{|c|c|c|c|c|c|c|}
\hline Total (c) & 0.340 & 0.050 & $<.001$ & $<0.001$ & 0.185 & 0.383 \\
\hline Total indirect & 0.181 & 0.027 & $\begin{array}{l}<.001 \\
0.00\end{array}$ & $<0.001$ & 0.100 & 0.206 \\
\hline
\end{tabular}

Specific indirect

$\begin{array}{lllllll}\text { COVID-19 perceived } & 0.038 & 0.012 & 0.009 & 0.014 & 0.012 & 0.060\end{array}$

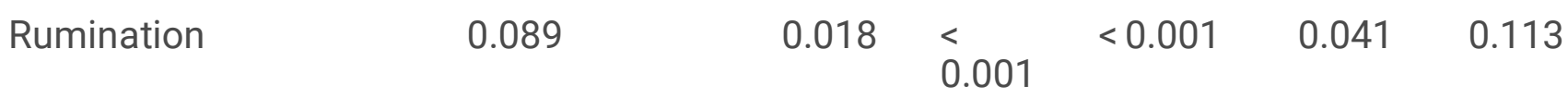

$\begin{array}{lllllll}\text { Attachment anxiety } & 0.005 & 0.005 & 0.474 & 0.511 & -0.006 & 0.016 \\ \text { Attachment avoidance } & 0.049 & 0.015 & 0.008 & 0.013 & 0.013 & 0.073 \\ \text { Direct }\left(c^{\prime}\right) & 0.159 & 0.050 & 0.007 & 0.012 & 0.037 & 0.228\end{array}$

\section{Anxiety}

$\begin{array}{llllllll}\text { Total }(\mathrm{c}) & 0.218 & 0.037 & 0.002 & 0.004 & 0.039 & 0.183 & 0.291 \\ \text { Total indirect } & 0.188 & 0.016 & < & <0.001 & 0.068 & 0.130 & \end{array}$

Specific indirect

$\begin{array}{lllllll}\text { COVID-19 perceived } & 0.049 & 0.009 & 0.005 & 0.009 & 0.011 & 0.047\end{array}$ stressors

$\begin{array}{lllllll}\text { Rumination } & 0.075 & 0.011 & < & 0.001 & 0.019 & 0.063\end{array}$

$\begin{array}{lllllll}\text { Attachment anxiety } & -0.008 & 0.005 & 0.392 & 0.433 & -0.015 & 0.003\end{array}$

$\begin{array}{lllllll}\text { Attachment avoidance } & 0.070 & 0.011 & < & 0.001 & 0.018 & 0.059\end{array}$

$\begin{array}{lllllll}\text { Direct (c') } & 0.030 & 0.036 & 0.658 & 0.674 & -0.057 & 0.084\end{array}$

\section{Stress}

Note. $\mathrm{DV}=$ Dependent Variable; $\mathrm{Cl}=$ Confidence Interval (bootstrapped); Std. = Standardised. Depicted are total, total indirect, specific indirect, and direct effects of the different multiple mediation models. $\mathrm{P}_{\text {corrected }}=$ false discovery rate corrected $\mathrm{p}$ value . 


\begin{tabular}{|c|c|c|c|c|c|c|c|}
\hline DV & $\begin{array}{l}\text { Std. point } \\
\text { estimate }\end{array}$ & SE & $\mathbf{p}$ & Pcorrected & $\begin{array}{l}\mathrm{Cl} \\
\text { Lower }\end{array}$ & $\begin{array}{l}\text { Cl } \\
\text { Upper }\end{array}$ & $\mathbf{R}^{2}$ \\
\hline Total (c) & 0.210 & 0.044 & $<.001$ & $<0.001$ & 0.070 & 0.243 & 0.347 \\
\hline Total indirect & 0.181 & 0.025 & $<_{0.001}$ & $<0.001$ & 0.088 & 0.186 & \\
\hline \multicolumn{8}{|l|}{ Specific indirect } \\
\hline $\begin{array}{l}\text { COVID-19 perceived } \\
\text { stressors }\end{array}$ & 0.045 & 0.012 & 0.005 & 0.009 & 0.014 & 0.061 & \\
\hline Rumination & 0.089 & 0.016 & $<.001$ & $<0.001$ & 0.038 & 0.101 & \\
\hline Attachment anxiety & 0.011 & 0.006 & 0.172 & 0.201 & -0.001 & 0.021 & \\
\hline Attachment avoidance & 0.036 & 0.013 & 0.044 & 0.057 & 0.001 & 0.055 & \\
\hline Direct $\left(c^{\prime}\right)$ & 0.029 & 0.044 & 0.615 & 0.646 & -0.065 & 0.107 & \\
\hline \multicolumn{8}{|l|}{ Loneliness } \\
\hline Total (c) & 0.425 & 0.003 & $<_{0.001}$ & $<0.001$ & 0.019 & 0.032 & 0.432 \\
\hline Total indirect & 0.190 & 0.002 & $<_{0.001}$ & $<0.001$ & 0.008 & 0.015 & \\
\hline \multicolumn{8}{|l|}{ Specific indirect } \\
\hline $\begin{array}{l}\text { COVID-19 perceived } \\
\text { stressors }\end{array}$ & 0.033 & 0.001 & 0.010 & 0.015 & 0.001 & 0.004 & \\
\hline Rumination & 0.049 & 0.001 & 0.003 & 0.006 & 0.001 & 0.005 & \\
\hline Attachment anxiety & 0.009 & $\begin{array}{l}< \\
0.001\end{array}$ & 0.204 & 0.231 & $<.001$ & 0.002 & \\
\hline Attachment avoidance & 0.100 & 0.001 & $<.001$ & $<0.001$ & 0.004 & 0.009 & \\
\hline Direct $\left(c^{\prime}\right)$ & 0.235 & 0.003 & $\begin{array}{l}< \\
0.001\end{array}$ & $<0.001$ & 0.008 & 0.02 & \\
\hline \multicolumn{8}{|l|}{ Paranoia } \\
\hline Total (c) & 0.314 & 0.054 & $<.001$ & $<0.001$ & 0.136 & 0.347 & 0.249 \\
\hline
\end{tabular}

Note. $\mathrm{DV}=$ Dependent Variable; $\mathrm{Cl}=$ Confidence Interval (bootstrapped); Std. = Standardised. Depicted are total, total indirect, specific indirect, and direct effects of the different multiple mediation models. $\mathrm{P}_{\text {corrected }}=$ false discovery rate corrected $\mathrm{p}$ value 


\begin{tabular}{|c|c|c|c|c|c|c|c|}
\hline DV & $\begin{array}{l}\text { Std. point } \\
\text { estimate }\end{array}$ & SE & $\mathrm{p}$ & Pcorrected & $\begin{array}{l}\mathrm{Cl} \\
\text { Lower }\end{array}$ & $\begin{array}{l}\text { Cl } \\
\text { Upper }\end{array}$ & $\mathrm{R}^{2}$ \\
\hline Total indirect & 0.118 & 0.021 & $<.001$ & $<0.001$ & 0.051 & 0.134 & \\
\hline \multicolumn{8}{|l|}{ Specific indirect } \\
\hline $\begin{array}{l}\text { COVID-19 perceived } \\
\text { stressors }\end{array}$ & 0.030 & 0.010 & 0.023 & 0.032 & 0.006 & 0.046 & \\
\hline Rumination & 0.033 & 0.013 & 0.054 & 0.067 & 0.001 & 0.053 & \\
\hline Attachment anxiety & 0.018 & 0.008 & 0.085 & 0.102 & 0.001 & 0.032 & \\
\hline Attachment avoidance & 0.038 & 0.015 & 0.047 & 0.060 & 0.001 & 0.059 & \\
\hline Direct $\left(c^{\prime}\right)$ & 0.196 & 0.053 & 0.004 & 0.008 & 0.046 & 0.255 & \\
\hline \multicolumn{8}{|l|}{$\begin{array}{l}\text { Psychological well- } \\
\text { being }\end{array}$} \\
\hline Total (c) & -0.292 & 0.024 & $<.001$ & $<0.001$ & -0.176 & -0.082 & 0.333 \\
\hline Total indirect & -0.186 & 0.014 & $<.001$ & $<0.001$ & -0.112 & -0.057 & \\
\hline \multicolumn{8}{|l|}{ Specific indirect } \\
\hline $\begin{array}{l}\text { COVID-19 perceived } \\
\text { stressors }\end{array}$ & -0.037 & 0.006 & 0.010 & 0.014 & -0.031 & -0.006 & \\
\hline Rumination & -0.102 & 0.011 & $<.001$ & $<0.001$ & -0.068 & -0.027 & \\
\hline Attachment anxiety & 0.004 & 0.003 & 0.555 & 0.583 & -0.004 & 0.008 & \\
\hline Attachment avoidance & -0.051 & 0.008 & 0.005 & 0.009 & -0.040 & -0.008 & \\
\hline Direct $\left(c^{\prime}\right)$ & -0.106 & 0.024 & 0.047 & 0.060 & -0.092 & 0.001 & \\
\hline \multicolumn{8}{|c|}{$\begin{array}{l}\text { Note. } \mathrm{DV}=\text { Dependent Variable; } \mathrm{Cl}=\text { Confidence Interval (bootstrapped); } \text { Std. = Standardised. Depicted } \\
\text { are total, total indirect, specific indirect, and direct effects of the different multiple mediation models. } \\
\mathrm{P}_{\text {corrected }}=\text { false discovery rate corrected } \mathrm{p} \text { value. }\end{array}$} \\
\hline
\end{tabular}

\section{Elastic Net Regression}

We explored the relative variable importance of different COVID-19 stressors in predicting psychosocial outcomes using elastic net regression and results from the selected models are depicted in Fig. 2. Of note, the selected elastic net regression model for outcome loneliness was ridge regression $(a=0)$, so none of the predictor variables were set exactly to zero, and LASSO $(a=1)$ for outcomes stress, paranoia, and wellbeing. The hyperparameter a was in between 0 to 1 for outcomes depression and anxiety 
reflecting the optimal balance between ridge and LASSO regression selected for these outcomes during cross-validation. Of note, analyses were adjusted for age and sex. See Supplementary Table 3 for hyperparameters of selected models.

A few COVID-19 stressors were rather consistently associated with adverse outcomes. Among them were "the current pandemic" (stressor 1), "worries of not being able to get medical care" (stressor 10), "increased conflicts with people close to me" (stressor 11), and "fears of what the future will bring, or that I won't be able to cope with everything" (stressor 14). In contrast, stressors such as "living in a small accommodation" (stressor 2), "being in home office" (stressor 7), and "uncertainties regarding my job, training place, studies or school" (stressor 13) were consistently unpredictive of adverse psychosocial outcomes. For some outcomes, the impact of COVID-19 perceived stressors was predominately associated with a few stressors that explained most of the variance. For example, levels of psychological well-being were only predicted by three COVID-19 stressors, namely "the current pandemic" (stressor 1), "increased conflicts with people close to me" (stressor 11), and "fears of what the future will bring, or that I won't be able to cope with everything" (stressor 14). For other psychosocial outcomes such as anxiety and loneliness, variable importance was rather equally distributed across stressors.

\section{Discussion}

This prospective study scrutinised the pathway between CM and multiple key psychosocial outcomes (depression, anxiety, stress, paranoia, loneliness, and psychological well-being) during the COVID-19 pandemic regarding potential mediators: 1) COVID-19 perceived stressors, 2) rumination and 3) insecure attachment while adjusting for important confounders of age, sex, income, educational attainment, and pre-existing mental health conditions. Our findings showed that COVID-19 perceived stressors may be a robust mechanism mediating the effect between $\mathrm{CM}$ and adverse psychosocial outcomes during the current pandemic. We also observed that rumination and insecure attachment (particularly attachment avoidance) acted as important additional mediators of the relationship between CM and psychosocial outcomes. The relative contribution of each potential mediator suggested that rumination was most strongly associated with adverse psychosocial outcomes. Of note, full mediation was observed for anxiety, stress and psychological well-being but not depression, paranoia, and loneliness. Taken together, our study adds to the accumulating research that highlights the importance of considering early adverse experiences when evaluating the psychological response to the current pandemic $(21-23,28,62)$. In addition, our findings suggest two important modifiable therapeutic targets, i.e. rumination and COVID-19 perceived stressors, the latter being specific to the pandemic for prevention and treatment efforts.

Our findings correspond well to the stress sensitisation hypothesis (27), which argues that early adverse experiences lead to stress sensitisation making individuals more reactive to later stressors and, thus, increasing the susceptibility for adult psychopathology. Indisputable, the current pandemic and associated countermeasures pose a heavy strain on many individuals with anxieties about the disease, 
temporary closures of educational institutes, enforced social isolation, job losses, financial difficulties to name only a few of the many stressors associated with the pandemic. Our findings show that the subjective perception of such COVID-19 related stressors matters in the relationship between CM and later adverse psychosocial outcomes during the pandemic. Moreover, we show that the impact of some stressors may be more relevant than others. Here, our explorative analyses highlight that perceived stress related to the pandemic itself, interpersonal conflicts, concerns about medical care resources, and worries about the future were among the stressors that mattered most for almost all adverse psychosocial outcomes. In sum, these results advance previous research on the relationship between CM and later adverse psychosocial outcomes by quantifying the relevance of perceived stressors specific to the pandemic (29).

For the outcomes of depression, loneliness, and paranoia, we observed only partial mediation suggesting that other mechanisms may be relevant to explain the relationship between early $\mathrm{CM}$ experiences and later adverse psychosocial outcomes. In our study, we focused on relatively established subjective psychological mediators of perceived stressors (specific to the COVID-19 pandemic), rumination, and insecure attachment. Yet, there may be other potentially relevant psychological but also biological mediators of the relationship that we did not assess and which may help to identify additional factors that are amenable to treatment. Psychologically, for instance, recent research has highlighted the importance of perceived lack of social support during the current pandemic (63) and its transdiagnostic importance has also been discussed before the pandemic (14). Biologically, proinflammatory processes have been implicated in depressive symptomology specifically $(64,65)$ and are proposed to explain the link between $\mathrm{CM}$ and later depression (66-68). As such, our study may have benefitted from inclusion of these factors and future longitudinal research should test their relative relevance in mediating the relationship between $\mathrm{CM}$ and later psychopathology in order to identify specific factors that can be therapeutically targeted.

\section{Strengths and limitations}

Strengths of the present study include i) the prospective study design, ii) assessment of perceived stressors specifically related to the COVID-19 pandemic, iii) simultaneous integration of established mediators of insecure attachment and rumination, iv) sophisticated analytical techniques, and v) inclusion of a broad array of key psychosocial outcomes. Yet, the study also has some important limitations. First, assessments are based exclusively on self-report questionnaires, so future research would benefit from inclusion of observer-based ratings, for example, by conducting structured interviews to assess attachment using the gold standard Adult Attachment Interview (69) or the Adult Attachment Projective (70). Second, although we report prospective data, only two timepoints of a 10-week time window were included and $\mathrm{CM}$ was assessed retrospectively. As several studies have shown a discrepancy between prospective and retrospective measures of $\mathrm{CM}(71,72)$, one has to be aware that $\mathrm{CM}$ scores always represent a subjective recall of adverse events during childhood which does not mean that this recall is invalid or less relevant. However, to minimise a potential recall bias, for example, due to current mood, we adjusted for self-reported life time mental health diagnoses. Yet, there may be other 
important confounders, for which we did not control. We also did not assess the specific forms, duration, or frequency of $\mathrm{CM}$, which may be important moderators but beyond the scope of the present study. Finally, we report data of an online survey unrepresentative of the German population, in which female sex and relatively young participants were overrepresented. Therefore, replication in more representative or cross-national samples would allow for greater generalisability of findings.

\section{Conclusion}

The current prospective study investigated the impact of $\mathrm{CM}$ on later adverse psychosocial outcomes during the COVID-19 pandemic. After adjusting for key confounding variable, we showed that subjective perception of COVID-19 stressors matters in the psychological response to the pandemic in addition to more established mediators of rumination and insecure attachment. Our findings underscore the importance of stress sensitisation in childhood trauma-exposed individuals that is also present in the current pandemic. Importantly, identified mediators of COVID-19 perceived stressors, rumination, and insecure attachment fully explain the relationship between $\mathrm{CM}$ and anxiety, stress or psychological wellbeing and are amenable to psychological interventions. Thus, researchers and clinicians who encounter patients with a history of $\mathrm{CM}$ during the pandemic are advised to assess these key psychological mediators for more targeted prevention and treatment efforts in order to prevent deterioration of mental health.

\section{Declarations}

\section{Acknowledgment}

We are grateful to individuals participating in the NEAR-Survey. We also thank Dr. Marcel Minke from Limesurvey-Consulting.com for the support in database set-up and data acquisition. This work was supported by the Network of Academic Medical Research into COVID-19 (NetzwerkUniversitätsmedizin NUM; grant number 01KX2021), funded by the Federal Ministry of Education and Research (BMBF).

\section{Conflicts of Interest}

Prof. Padberg reports personal fees and non-financial support from Mag \& More GmbH, Munich, Germany, personal fees and non-financial support from Brainsway Inc., Jerusalem, Israel, personal fees and non-financial support from neuroConn $\mathrm{GmbH}$, Ilmenau, Germany, outside the submitted work. The other authors declare no competing interests.

\section{Author contributions}

SR, MRand FP contributed to the study conception and design. Material preparation, data collection and analysis were performed by SR, MB and FP. The first draft of the manuscript was written by SR and all 
authors commented on previous versions of the manuscript. All authors read and approved the final manuscript.

\section{Availability of data and materials}

The datasets used and/or analysed during the current study are available from the corresponding author on reasonable request.

\section{References}

1. Holmes EA, O'Connor RC, Perry VH, Tracey I, Wessely S, Arseneault L, et al. Multidisciplinary research priorities for the COVID-19 pandemic: a call for action for mental health science. The Lancet Psychiatry. 2020;547-60.

2. Rek S V, Freeman D, Reinhard MA, Bühner M, Grosen S, Falkai P, et al. Differential psychological response to the COVID - 19 pandemic in psychiatric inpatients compared to a non - clinical population from Germany. Eur Arch Psychiatry Clin Neurosci [Internet]. 2021; Available from: https://doi.org/10.1007/s00406-021-01291-7

3. Pierce M, Hope H, Ford T, Hatch S, Hotopf M, John A, et al. Mental health before and during the COVID-19 pandemic: a longitudinal probability sample survey of the UK population. The Lancet Psychiatry. 2020;7(10):883-92.

4. Shanahan L, Steinhoff A, Bechtiger L, Murray AL, Nivette A, Hepp U, et al. Emotional distress in young adults during the COVID-19 pandemic: Evidence of risk and resilience from a longitudinal cohort study. Psychol Med. 2020;1-10.

5. Robinson E, Sutin AR, Daly M, Jones A. A systematic review and meta-analysis of longitudinal cohort studies comparing mental health before versus during the COVID-19 pandemic. medRxiv. 2021;

6. Shevlin M, Butter S, McBride O, Murphy J, Gibson-Miller J, Hartman TK, et al. Refuting the myth of a 'tsunami'of mental ill-health in populations affected by COVID-19: Evidence that response to the pandemic is heterogenous, not homogeneous. Psychol Med. 2021;1-30.

7. Luchetti M, Lee JH, Aschwanden D, Sesker A, Strickhouser JE, Terracciano A, et al. The trajectory of Ioneliness in response to COVID-19. Am Psychol. 2020;

8. Bu F, Steptoe A, Fancourt D. Loneliness during a strict lockdown: Trajectories and predictors during the COVID-19 pandemic in 38,217 United Kingdom adults. Soc Sci Med. 2020;265:113521.

9. Fancourt D, Steptoe A, Bu F. Trajectories of anxiety and depressive symptoms during enforced isolation due to COVID-19 in England: a longitudinal observational study. The Lancet Psychiatry. 2021;8(2):141-9.

10. Saunders R, Buckman JEJ, Fonagy P, Fancourt D. Understanding different trajectories of mental health across the general population during the COVID-19 pandemic. Psychol Med. 2021;1-9.

11. van Zyl LE, Rothmann S, Zondervan-Zwijnenburg MAJ. Longitudinal trajectories of study characteristics and mental health before and during the COVID-19 lockdown. Front Psychol. 2021;12. 
12. Iob E, Frank P, Steptoe A, Fancourt D. Levels of severity of depressive symptoms among at-risk groups in the UK during the COVID-19 pandemic. JAMA Netw open. 2020;3(10):e2026064e2026064.

13. Keyes KM, Eaton NR, Krueger RF, McLaughlin KA, Wall MM, Grant BF, et al. Childhood maltreatment and the structure of common psychiatric disorders. Br J Psychiatry. 2012;200(2):107-15.

14. McLaughlin KA, Colich NL, Rodman AM, Weissman DG. Mechanisms linking childhood trauma exposure and psychopathology: a transdiagnostic model of risk and resilience. BMC Med. 2020;18:1-11.

15. Nelson CA, Scott RD, Bhutta ZA, Harris NB, Danese A, Samara M. Adversity in childhood is linked to mental and physical health throughout life. bmj. 2020;371.

16. Albott CS, Forbes MK, Anker JJ. Association of childhood adversity with differential susceptibility of transdiagnostic psychopathology to environmental stress in adulthood. JAMA Netw open. 2018;1(7):e185354-e185354.

17. Li M, D’arcy C, Meng X. Maltreatment in childhood substantially increases the risk of adult depression and anxiety in prospective cohort studies: systematic review, meta-analysis, and proportional attributable fractions. Psychol Med. 2016;46(4):717-30.

18. Varese F, Smeets F, Drukker M, Lieverse R, Lataster T, Viechtbauer W, et al. Childhood adversities increase the risk of psychosis: a meta-analysis of patient-control, prospective-and cross-sectional cohort studies. Schizophr Bull. 2012;38(4):661-71.

19. Hughes K, Lowey H, Quigg Z, Bellis MA. Relationships between adverse childhood experiences and adult mental well-being: results from an English national household survey. BMC Public Health. 2016;16(1):1-11.

20. Chi X, Becker B, Yu Q, Willeit P, Jiao C, Huang L, et al. Prevalence and psychosocial correlates of mental health outcomes among chinese college students during the coronavirus disease (covid-19) pandemic. Front psychiatry. 2020;11:803.

21. Lahav Y. Psychological distress related to COVID-19-the contribution of continuous traumatic stress. J Affect Disord. 2020;277:129-37.

22. Tsur N, Abu-Raiya H. COVID-19-related fear and stress among individuals who experienced child abuse: The mediating effect of complex posttraumatic stress disorder. Child Abuse Negl. 2020;110:104694.

23. Guo J, Fu M, Liu D, Zhang B, Wang X, van IJzendoorn MH. Is the psychological impact of exposure to COVID-19 stronger in adolescents with pre-pandemic maltreatment experiences? A survey of rural Chinese adolescents. Child Abuse Negl. 2020;110:104667.

24. Kim AW, Nyengerai T, Mendenhall E. Evaluating the mental health impacts of the COVID-19 pandemic: perceived risk of COVID-19 infection and childhood trauma predict adult depressive symptoms in urban South Africa. Psychol Med. 2020;1-13.

25. Heim CM, Entringer S, Buss C. Translating basic research knowledge on the biological embedding of early-life stress into novel approaches for the developmental programming of lifelong health. 
Psychoneuroendocrinology. 2019;105:123-37.

26. Tiwari A, Gonzalez A. Biological alterations affecting risk of adult psychopathology following childhood trauma: a review of sex differences. Clin Psychol Rev. 2018;66:69-79.

27. Hammen C, Henry R, Daley SE. Depression and sensitization to stressors among young women as a function of childhood adversity. J Consult Clin Psychol. 2000;68(5):782.

28. Berens AE, Jensen SKG, Nelson CA. Biological embedding of childhood adversity: from physiological mechanisms to clinical implications. BMC Med. 2017;15(1):1-12.

29. Gotlib IH, Borchers LR, Chahal R, Gifuni AJ, Ho T. Early Life Stress Predicts Depressive Symptoms in Adolescents During the COVID-19 Pandemic: The Mediating Role of Perceived Stress. Available SSRN 3606441. 2020;

30. Weissman DG, Bitran D, Miller AB, Schaefer JD, Sheridan MA, McLaughlin KA. Difficulties with emotion regulation as a transdiagnostic mechanism linking child maltreatment with the emergence of psychopathology. Dev Psychopathol. 2019;31(3):899.

31. Mansueto G, Cavallo C, Palmieri S, Ruggiero GM, Sassaroli S, Caselli G. Adverse childhood experiences and repetitive negative thinking in adulthood: a systematic review. Clin Psychol Psychother. 2021;

32. LeMoult J, Humphreys KL, King LS, Colich NL, Price AN, Ordaz SJ, et al. Associations among early life stress, rumination, symptoms of psychopathology, and sex in youth in the early stages of puberty: A moderated mediation analysis. J Abnorm Child Psychol. 2019;47(2):199-207.

33. Ein-Dor T, Viglin D, Doron G. Extending the transdiagnostic model of attachment and psychopathology. Front Psychol. 2016;7:484.

34. Healy C, Eaton A, Cotter I, Carter E, Dhondt N, Cannon M. Mediators of the longitudinal relationship between childhood adversity and late adolescent psychopathology. Psychol Med. 2021;1-9.

35. Association GA of the WM. World Medical Association Declaration of Helsinki: ethical principles for medical research involving human subjects. J Am Coll Dent. 2014;81(3):14.

36. Bernstein DP, Fink L, Handelsman L, Foote J. Childhood trauma questionnaire. Assess Fam violence A Handb Res Pract. 1998;

37. Klinitzke G, Romppel M, Häuser W, Brähler E, Glaesmer H. The German Version of the Childhood Trauma Questionnaire (CTQ): psychometric characteristics in a representative sample of the general population. Psychother Psychosom Med Psychol. 2011;62(2):47-51.

38. Rek SV, Freeman D, Reinhard M, Bühner M, Keeser D, Padberg F. The COVID-19 Pandemic Mental Health Questionnaire (CoPaQ): Introducing a comprehensive measure of the psychosocial impact of the current coronavirus crisis. Open Sci Framew. 2020;

39. Rek S V, Bühner M, Reinhard MA, Freeman D, Keeser D, Adorjan K, et al. The COVID-19 Pandemic Mental Health Questionnaire (CoPaQ): Psychometric Evaluation and Compliance with Countermeasures in Psychiatric Inpatients and Non-clinical Individuals. 2021; 
40. Ehring T, Zetsche U, Weidacker K, Wahl K, Schönfeld S, Ehlers A. The Perseverative Thinking Questionnaire (PTQ): Validation of a content-independent measure of repetitive negative thinking. J Behav Ther Exp Psychiatry. 2011;42(2):225-32.

41. Griffin DW, Bartholomew K. The metaphysics of measurement: The case of adult attachment. 1994;

42. Steffanowski A, Oppl M, Meyerberg J, Schmidt J, Wittmann WW, Nübling R. Psychometrische Überprüfung einer deutschsprachigen version des relationship scales questionnaire (RSQ). Störungsspezifische Ther der Station Psychother Giess Psychosoz Verlag. 2001;320-42.

43. Roisman GI, Holland A, Fortuna K, Fraley RC, Clausell E, Clarke A. The Adult Attachment Interview and self-reports of attachment style: an empirical rapprochement. J Pers Soc Psychol. 2007;92(4):678.

44. Henry JD, Crawford JR. The short-form version of the Depression Anxiety Stress Scales (DASS-21): Construct validity and normative data in a large non-clinical sample. Br J Clin Psychol. 2005;44(2):227-39.

45. Nilges P, Essau C. Die Depressions-Angst-Stress-Skalen. Der Schmerz. 2015;29(6):649-57.

46. Antony MM, Cox BJ, Enns MW, Bieling PJ, Swinson RP. Psychometric properties of the 42-item and 21-item versions of the Depression Anxiety Stress Scales in clinical groups and a community sample. Psychol Assess. 1998;10(2):176-81.

47. Freeman D, Loe BS, Kingdon D, Startup H, Molodynski A, Rosebrock L, et al. The revised Green et al. , Paranoid Thoughts Scale (R-GPTS): psychometric properties, severity ranges, and clinical cut-offs . Psychol Med. 2019;1-10.

48. Rek SV, Freeman D, Reinhard M, Bühner M, Padberg F. Psychometric evaluation of the German Revised-Green et al. Paranoid Thought Scales (R-GPTS) across clinical and non-clinical samples. Prep. 2021;

49. Brislin RW. Back-translation for cross-cultural research. J Cross Cult Psychol. 1970;1(3):185-216.

50. Russell D, Peplau LA, Cutrona CE. The revised UCLA Loneliness Scale: Concurrent and discriminant validity evidence. J Pers Soc Psychol. 1980;39(3):472-80.

51. Döring N, Bortz J. Psychometrische Einsamkeitsforschung: Deutsche Neukonstruktion der UCLA Loneliness Scale. Diagnostica. 1993;

52. Organization WH. Wellbeing measures in primary health care/the DEPCARE project: report on a WHO meeting, Stockholm, Sweden 12-13 February 1998. In: Wellbeing measures in primary health care/the DEPCARE project: report on a WHO meeting, Stockholm, Sweden 12-13 February 1998. 1998.

53. Brähler E, Mühlan H, Albani C, Schmidt S. Teststatistische prüfung und normierung der deutschen versionen des EUROHIS-QOL lebensqualität-Index und des WHO-5 wohlbefindens-index. Diagnostica. 2007;53(2):83-96.

54. Topp CW, Østergaard SD, Søndergaard S, Bech P. The WHO-5 Well-Being Index: a systematic review of the literature. Psychother Psychosom. 2015;84(3):167-76.

55. Team RC. R: A language and environment for statistical computing. 2013;

56. Revelle W, Revelle MW. Package 'psych.' Compr R Arch Netw. 2015; 
57. Rosseel Y. Lavaan: An R package for structural equation modeling and more. Version 0.5-12 (BETA). J Stat Softw. 2012;48(2):1-36.

58. Friedman J, Hastie T, Tibshirani R. Regularization paths for generalized linear models via coordinate descent. J Stat Softw. 2010;33(1):1.

59. Thissen D, Steinberg L, Kuang D. Quick and easy implementation of the Benjamini-Hochberg procedure for controlling the false positive rate in multiple comparisons. J Educ Behav Stat. 2002;27(1):77-83.

60. Zou H, Hastie T. Regularization and variable selection via the elastic net. J R Stat Soc Ser B (statistical Methodol. 2005;67(2):301-20.

61. Tibshirani R. Regression shrinkage and selection via the lasso. J R Stat Soc Ser B. 1996;58(1):26788.

62. Hoerl AE, Kennard RW. Ridge regression: Biased estimation for nonorthogonal problems. Technometrics. 1970;12(1):55-67.

63. Seitz KI, Bertsch K, Herpertz SC. A Prospective Study of Mental Health During the COVID-19 Pandemic in Childhood Trauma-Exposed Individuals: Social Support Matters. J Trauma Stress. 2021;

64. Kappelmann N, Lewis G, Dantzer R, Jones PB, Khandaker GM. Antidepressant activity of anticytokine treatment: a systematic review and meta-analysis of clinical trials of chronic inflammatory conditions. Mol Psychiatry. 2018;23(2):335-43.

65. Milaneschi Y, Kappelmann N, Ye Z, Lamers F, Moser S, Jones PB, et al. Association of Inflammation with Depression and Anxiety: Evidence for Symptom-Specificity and Potential Causality from UK Biobank and NESDA Cohorts. medRxiv. 2021;

66. Baumeister D, Akhtar R, Ciufolini S, Pariante CM, Mondelli V. Childhood trauma and adulthood inflammation: a meta-analysis of peripheral C-reactive protein, interleukin- 6 and tumour necrosis factor-a. Mol Psychiatry. 2016;21(5):642-9.

67. Slavich GM, Irwin MR. From stress to inflammation and major depressive disorder: a social signal transduction theory of depression. Psychol Bull. 2014;140(3):774.

68. Nemeroff CB. The state of our understanding of the pathophysiology and optimal treatment of depression: Glass half full or half empty? Am J Psychiatry. 2020;177(8):671-85.

69. George C, Kaplan N, Main M. Attachment interview for adults. Unpubl manuscript, Univ California, berkeley. 1985;

70. Bauriedl-Schmidt C, Jobst A, Gander M, Seidl E, Sabaß L, Sarubin N, et al. Attachment representations, patterns of emotion regulation, and social exclusion in patients with chronic and episodic depression and healthy controls. J Affect Disord. 2017;210:130-8.

71. Baldwin JR, Reuben A, Newbury JB, Danese A. Agreement Between Prospective and Retrospective Measures of Childhood Maltreatment A Systematic Review and Meta-analysis. 2019;76(6):584-93. 
72. Danese A, Widom CS. Objective and subjective experiences of child maltreatment and their relationships with psychopathology. Nat Hum Behav. 2020;4(8):811-8.

\section{Figures}
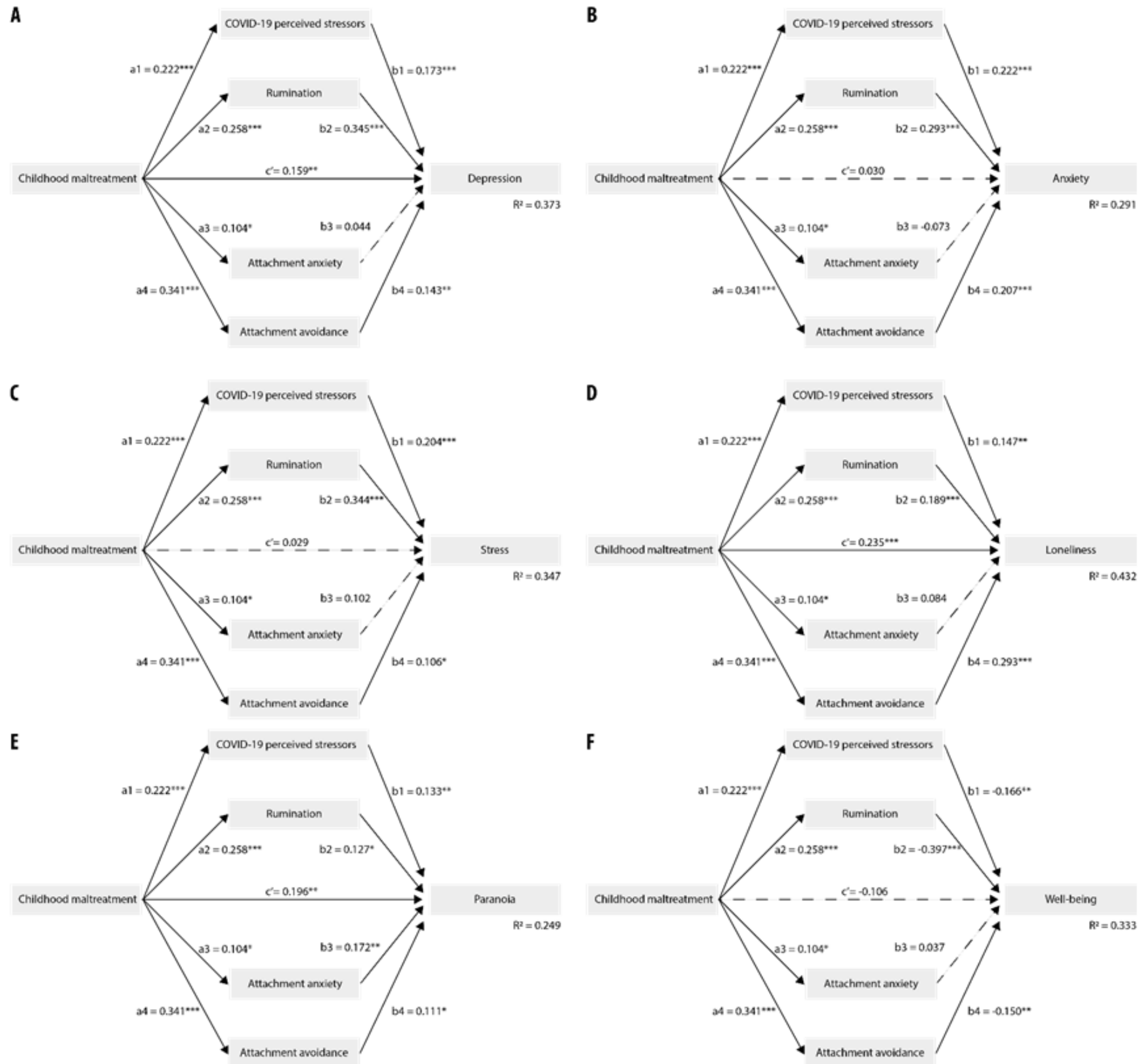

\section{Figure 1}

Multiple mediation models Note. The figure shows path diagrams for multiple mediation models for outcomes depression (A), anxiety (B), stress (C), loneliness (D), paranoia (E), and psychological well-being (F). Non-significant paths are visualised using dashed lines. Regression coefficients are standardised. 

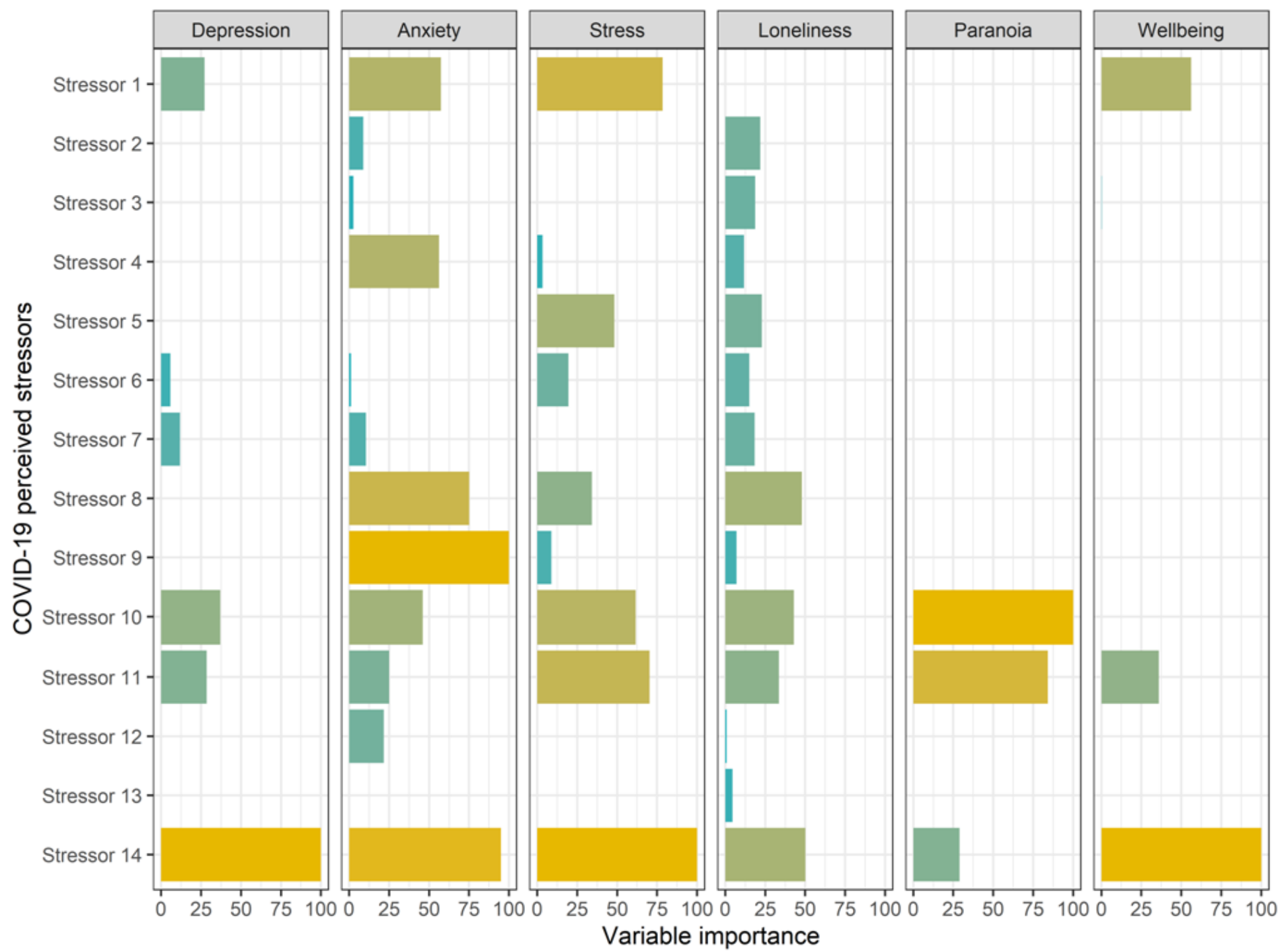

\section{Figure 2}

Relative importance of COVID-19 related stressors in predicting psychosocial outcomes Note. Relative importance was extracted from elastic net regression models with optimal hyperparameters selected during cross-validation (see Supplementary Table 1). Analyses were adjusted for age and sex. Stressor 1 = the current pandemic, Stressor 2 = living in a small accommodation, Stressor 3 = being in quarantine, Stressor 4 = childcare, Stressor $5=$ taking over school lessons, Stressor $6=$ the curfew, Stressor $7=$ being in home office, Stressor 8 = customer service, Stressor 9 = worries about my health, Stressor $10=$ worries of not being able to get medical care, Stressor $11=$ increased conflicts with people close to me, Stressor 12 = financial worries, Stressor 13 = uncertainties regarding my job, training place, studies or school, Stressor14 $=$ fears of what the future will bring, or that I won't be able to cope with everything.

\section{Supplementary Files}

This is a list of supplementary files associated with this preprint. Click to download. 
- 20210722SupplementaryMaterial.docx 